\title{
Characterisation of sleep in intensive care using 24-hour polysomnography: an observational study
}

Rosalind Elliott ${ }^{1 *}$, Sharon McKinley ${ }^{1,2}$, Peter Cistulli, ${ }^{3,4}$ and Mary Fien ${ }^{5}$

\begin{abstract}
Introduction: Many intensive care patients experience sleep disruption potentially related to noise, light and treatment interventions. The purpose of this study was to characterise, in terms of quantity and quality, the sleep of intensive care patients, taking into account the impact of environmental factors.

Methods: This observational study was conducted in the adult ICU of a tertiary referral hospital in Australia, enrolling 57 patients. Polysomnography (PSG) was performed over a 24-hour period to assess the quantity (total sleep time: hh:mm) and quality (percentage per stage, duration of sleep episode) of patients' sleep while in ICU. Rechtschaffen and Kales criteria were used to categorise sleep. Interrater checks were performed. Sound pressure and illuminance levels and care events were simultaneously recorded. Patients reported on their sleep quality in ICU using the Richards Campbell Sleep Questionnaire and the Sleep in Intensive Care Questionnaire. Data were summarised using frequencies and proportions or measures of central tendency and dispersion as appropriate and Cohen's Kappa statistic was used for interrater reliability of the sleep data analysis.

Results: Patients' median total sleep time was 05:00 (IQR: 02:52 to 07:14). The majority of sleep was stage 1 and 2 (medians: 19 and 73\%) with scant slow wave and REM sleep. The median duration of sleep without waking was 00:03. Sound levels were high (mean Leq $53.95 \mathrm{~dB}(\mathrm{~A})$ during the day and $50.20 \mathrm{~dB}(\mathrm{~A})$ at night) and illuminance levels were appropriate at night (median <2 lux) but low during the day (median: 74.20 lux). There was a median 1.7 care events/h. Patients' mean self-reported sleep quality was poor. Interrater reliability of sleep staging was highest for slow wave sleep and lowest for stage 1 sleep.

Conclusions: The quantity and quality of sleep in intensive care patients are poor and may be related to noise, critical illness itself and treatment events that disturb sleep. The study highlights the challenge of quantifying sleep in the critical care setting and the need for alternative methods of measuring sleep. The results suggest that a sound reduction program is required and other interventions to improve clinical practices to promote sleep in intensive care patients.
\end{abstract}

Trial registration: Australian New Zealand clinical trial registry (http://www.anzctr.org.au/): ACTRN12610000688088.

\section{Introduction}

The role of good quality sleep in health is well known $[1,2]$. However, many critically ill patients treated in the intensive care unit (ICU) experience sleep disruption [3-6]. Intensive care patients may experience normal or nearnormal total sleep time (TST) but have been reported to have reduced slow wave (SW) and rapid eye movement (REM) sleep, largely because of frequent arousals. The factors causing sleep disruption in intensive care patients are

\footnotetext{
* Correspondence: Rosalind.Elliott@uts.edu.au

'Faculty of Health, University of Technology Sydney, 235-253 Jones Street, Broadway 2007, New South Wales, Australia

Full list of author information is available at the end of the article
}

not fully understood; many extrinsic and intrinsic factors have been proposed, such as noise [7], light, inflammatory mediators [6], sedative and opioid medications [4] and mechanical ventilator settings [8]. Polysomnography (PSG) recording is supported by reports from ICU patients on their inability to sleep well in ICU $[9,10]$. Patients often attribute disturbances to their sleep to noise levels [10].

Twenty-four hour PSG studies in ICU have been few and there are no recent studies; there have been fewer studies still examining the prevalence of sleep disruptive factors simultaneously with PSG or investigations of ICU patient perceptions of the quality of sleep and sleep disruptions in conjunction with PSG recording. The primary aim of this

\section{C) Biomed Central}


observational study was to provide a current assessment of the quality and quantity of critically ill patients' sleep while they were treated in ICU. The secondary aims were to explore the prevalence of the main factors that potentially affect sleep in ICU, that is the environmental sound pressure and illuminance levels ICU patients are exposed to, and the frequency of treatment and care patients receive. We also assessed self-reported sleep quality while patients were treated in ICU and the hospital ward and patients' perceptions of sleep disruptions in ICU.

\section{Materials and methods Study setting and sample}

This study was conducted in a 36-bed adult general ICU in a 600-bed hospital in Sydney, Australia. The ICU provided specialty services such as cardiac, spinal, burns and renal and was a closed unit with an accredited intensivist responsible for the management of all patients. Ward rounds were conducted by the intensivist twice a day when treatment goals/plans were reviewed, for example sedation medication prescriptions were adjusted according to the individual patient needs (routinely, calm and interactive). The registered nurse (RN) to patient ratio was 1:1 for mechanically ventilated patients and 1:2 for patients requiring high dependency care. There was no distinct area for the care of high dependency level patients; mechanically ventilated patients were cared for alongside patients of lower acuity. The RN performed all the nursing care for the patient. The main practices associated with sleep promotion during the time in which the study was conducted were offering a night-time hypnotic (that is, temazepam) and earplugs/eye shades to the patients and dimming the main lights at night.

Patients were included if aged $>16$ years and likely to be treated in ICU for $>24$ hours and able to give informed consent on their own behalf. The ability to provide consent was assessed by the following means: i) subjective advice by the bedside nurse; cognition was discussed including orientation to time and place and ability to follow simple instructions; ii) the patient was approached and asked to state their name (or mouth the words); if the patient was able to perform this instruction a further check was made; iii) the patient's understanding and ability to follow instructions was checked by asking them to nod when the correct colour card was held up from a selection of three. Exclusion criteria included a history of sleep disorders, psychiatric illness requiring medication, a known diagnosis of dementia or central neurological impairment confirmed by radiological scan. Human Research Ethics Committee approval for the study was provided by the Health Service and the University of Technology Sydney. Patients provided informed consent with written confirmation by their next of kin in cases where the patient was unable to sign the consent form. Data were collected from
January to December, 2009 and September 2010 to April 2011. Screening for eligibility was performed on weekdays.

\section{Data collection}

On enrolment, patients rated their sleep quality prior to hospitalization using a 1 to 10 scale $(10=$ excellent $)$ and patients or their proxy completed the Insomnia Severity Index (ISI) [11]. The ISI comprises seven items based on the symptoms and severity of sleep disturbance; each item is scored from 0 (not at all) to 4 (extremely). The total ISI score ranges from 0 to 28 with a cutoff for clinical insomnia of 15 . Concurrent validity with a sleep diary has been reported at $\mathrm{r}=0.65$ [11].

Patients were monitored for one 24-hour period using a portable PSG device, either PS-2 ${ }^{\mathrm{TM}}$ (Compumedics, Melbourne, Australia) or ALICE LE ${ }^{\mathrm{TM}}$ (Philips Respironics, Amsterdam, Netherlands). Recording began and finished between 1000 and 1700 hours. Electroencephalograph (EEG) (O1/M2, C4/M1), electromyograph (EMG), electrooculograph (EOG) (right and left) and electrocardiograph (ECG) (lead II) were recorded. Patients' skin was prepared according to standard techniques. Gold cup EEG electrodes were placed at O1/M2 and C4/M1 according to the International 10-20 System [12]. Two EOG electrodes were used for right and left eye movements. The EMG electrodes were located over the right and left masseter (facial) muscles. Electrode application was performed by the authors (RE and MF) who were both trained in the technique. Electrode impedance was maintained $<9,000$ ohms. Visual checks were performed hourly and electrodes were replaced if impedance values approached 9,000 ohms or when the patient was disturbed for routine repositioning.

Sound and illuminance levels were recorded simultaneously with PSG using the integrated sound pressure level meter (model 2250, Brüel and KjaerTM, Nærum, Denmark) and illuminance level meter (T-10, Konica Minolta $^{\mathrm{TM}}$, Osaka, Japan). Continuous equivalent sound pressure levels (Leq) in ' $\mathrm{A}$ ' weighted decibels and peak sound pressure levels (Lpeak) in ' $C$ ' weighted decibels were logged every second. More detailed information about the protocol for sound pressure level recording and configuration of patient rooms is reported elsewhere [13]. Illuminance level (in lux) was recorded, using a sensor placed close to the patient's head, once per minute. The bedside nurse was requested to log an event whenever the patient received treatment or care using a specially designed Microsoft Access ${ }^{\mathrm{TM}}$ (Microsoft, Redmond, WA, USA) form listing a number of events on a computer located within reach. The event log contained the following items: clinical assessment; tracheal suctioning; pressure area care; physiotherapy; mouth/eye care; blood test (sampling); wash; non-invasive blood pressure; eating and drinking; dressing; pain; line insertion; X-ray; clinical 
crisis; agitation/anxiety/confusion; electrode replacement and other (for example placing an extra blanket on the patient).

On completion of PSG recording patients rated their previous night's sleep using the Richards Campbell Sleep Questionnaire (RCSQ) [14]. The RCSQ contains five $100 \mathrm{~mm}$ visual analogue scales (VAS): sleep depth, latency, awakenings, time awake and quality of sleep (higher scores indicate better sleep). The RCSQ was pilot tested in a medical ICU [15] and validated with overnight PSG in medical ICU patients [14]. In our study, patients who were unable to write were assisted; the patient used their current communication strategy to indicate where the investigator should mark the VAS.

One to two nights after transfer to the ward, patients rated their sleep on the ward using the RCSQ and reported on sleep-disturbing factors in ICU using the Sleep in Intensive Care Questionnaire (SICQ) [10]. The SICQ was developed to determine the perceived effect of the ICU environment on sleep. It contains seven questions (rated 1 to 10), including overall sleep quality at home, sources of perceived sleep disruption and sources of noise.

Demographic and clinical data were collected from the patient's record. The Acute Physiology and Chronic Health Evaluation (APACHE) III modified diagnostic codes [16] were used to classify diagnoses. The APACHE II severity of illness score on admission and the Modified Sequential Organ Failure Assessment (SOFA) [17] at the time of enrolment were calculated to assess severity of illness.

\section{Data analysis}

Polysomnography recordings were scored manually in 30second epochs, by three qualified sleep technologists using standard Rechtschaffen and Kales [18] (R and K) criteria. TST was defined as the time spent in all sleep stages. The percentage of time in each sleep stage and sleep during the day (0600 to 2100 hours) was calculated. Arousals were defined using American Academy of Sleep Medicine criteria [19] and the number per hour of sleep (arousal index) reported. Morphine equivalent doses of opioid medications [20] and midazolam equivalent doses of benzodiazepine [21] were calculated to summarise the doses of opioid and sedative medications administered.

The software packages PASWTM (version 18; SPSS Inc, Chicago, IL, USA) and Microsoft Excel (2007) were used to analyse the data. Means and medians were used to describe continuous data and frequencies and percentages were used for categorical data. Interrater reliability for scoring the PSG data by sleep technologists was performed on 16 (30\%) recordings using Cohen's Kappa statistic. The Mann-Whitney U test was used to detect differences between the TST and arousal indices for patients who received mechanical ventilation during PSG and those who did not and to compare arousal indices for patients who received benzodiazepine medications or propofol and those who did not. The correlation between arousal indices and peak sounds ( $>80 \mathrm{~dB}(\mathrm{~A})$ ) was explored using Pearson's $r$. Wilcoxon signed ranks test was used to compare patients' self-reported quality of sleep in ICU and the ward.

\section{Results}

\section{Sample characteristics}

During the study twenty-four hour PSG data were analysed for 53 patients and 47 patients were followed up on the ward. Of 266 eligible patients 57 were enrolled; of those not enrolled 74 declined participation and 135 were transferred to the ward before they could be invited to participate. Two patients requested removal of PSG after recording began, palliation was initiated for another and data for another was unable to be analysed. Figure 1 provides a flow diagram of the number of eligible patients and the number invited to participate and enrolled. The characteristics of patients enrolled in the study are provided in Table 1. Admission diagnoses were mostly non-operative (66\%), mean APACHE II score was 18.70 (SD: 8.23) and the mean SOFA score was 4.04 (SD: 2.53). Patients were interactive and calm (mean Vancouver Interaction Scale (VICS) [22] score: 27.06 (SD: 3.80) (equivalent to 0 or -1 on the Richmond Agitation Sedation Scale (RASS) [23]).

Twenty-one percent of patients reported a pre-hospital ISI score of $\geq 15$, indicating moderate to severe clinical insomnia, however, median pre-hospitalisation sleep quality on the SICQ was 8.00 out of 10.00 . There was no difference between the mean RCSQ score in ICU and on the ward $(P=0.61)$. Noise was rated the highest sleepdisturbing factor (Table 2).

\section{Sleep, sound and light}

Median TST was five hours (Figure 2). The median duration of sleep without waking was 00:03 (hh:mm). Unusual sleep stage transitions were noted (for example progression from stage 1 to REM sleep). Daytime sleep comprised $41 \%$ of TST and EEG delta wave activity was observed when patients were interactive and apparently awake. The majority of sleep was stages 1 and 2 (19 and $73 \%$, respectively). SW and REM sleep was scant (median (IQR) 0 (0 to 1 ) and 0 (0 to 6) respectively). Arousals were frequent (median arousal index: 27) (Table 3). Interrater reliability of the sleep technologists' PSG analysis for sleep/awake was 0.58 to 0.68 (Table 4 ).

Sound pressure levels were high (mean Leq 53.95 (SD: 2.33) $\mathrm{dB}(\mathrm{A})$ during the day and 50.20 (SD: 3.21) $\mathrm{dB}(\mathrm{A})$ at night). There were a median $416 / \mathrm{h}$ sound peaks $>80$ $\mathrm{dB}(\mathrm{C})$ during the day and $90 / \mathrm{h}$ at night. The correlation between arousal indices and number of sound peaks $>80 \mathrm{~dB}(\mathrm{~A})$ was weak during the day $(\mathrm{r}=0.13)$ and night 


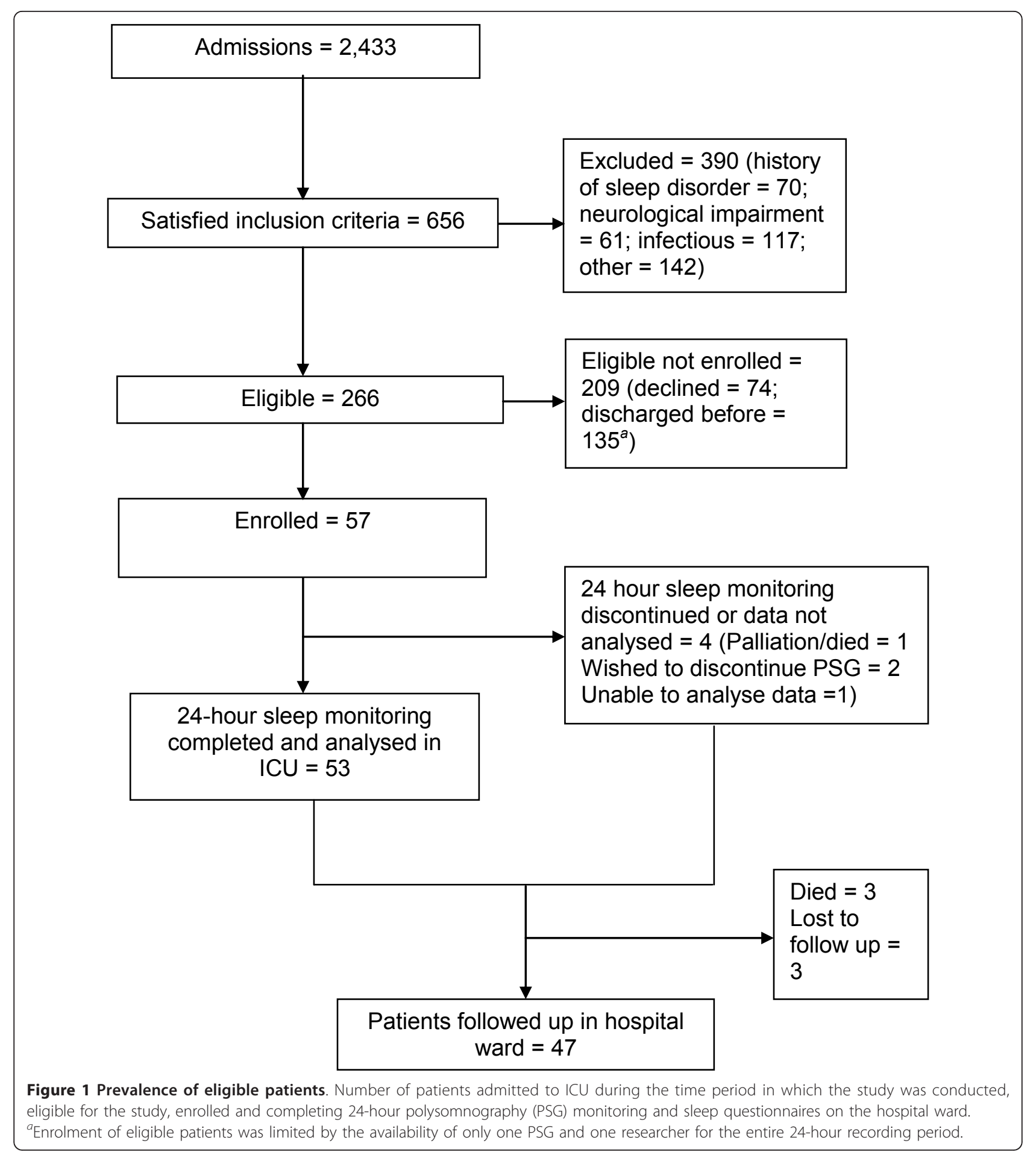

$(r=0.19)$; neither was statistically significant. Median illuminance levels were lower at night (1.74 lux) than during the day (74.20 lux). The average number of care events was $1.74 / \mathrm{h}$; the lowest was between 0200 and 0500 hours $(<1.00 / \mathrm{h})$. No patients wore ear plugs or eye shades during sleep monitoring.

\section{Sleep mechanical ventilation and medications}

Pressure support was the ventilation mode used for 26 patients (six patients received pressure control ventilation (PCV) for periods during pressure support), one patient received synchronised intermittent ventilation (SIMV) and another patient received PCV. Twelve 
Table 1 Patient characteristics

\begin{tabular}{|c|c|}
\hline Characteristics & $n=53$ \\
\hline Females, n (\%) & $17(32)$ \\
\hline \multicolumn{2}{|l|}{ Diagnosis, n (\%) } \\
\hline Operative & $18(34)$ \\
\hline Non-operative & $35(66)$ \\
\hline Age, mean $(S D)^{a}, y$ & $60.13(20.02)$ \\
\hline APACHE ${ }^{b} \|$ score, mean (SD) & $18.70(8.23)$ \\
\hline SOFA'score, mean (SD) & $4.04(2.53)$ \\
\hline \multicolumn{2}{|l|}{ Sedation level on enrolment $\left(\mathrm{VICS}^{d}\right)$, mean (SD) } \\
\hline Interaction score & $27.06(3.80)$ \\
\hline Calmness score & $29.00(2.70)$ \\
\hline 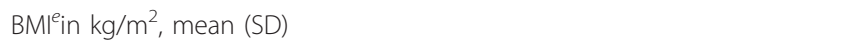 & $24.50(4.90)$ \\
\hline Duration of ventilation, median $\left(I Q R^{f}\right), d$ & $6.00(1.67-21.50)$ \\
\hline Length of ICU stay, median (IQR), d & $12.00(6.00-26.00)$ \\
\hline Length of hospital, median (IQR), d & $29.00(17.50-49.50)$ \\
\hline ICU admission day on which sleep monitoring occurred, median (IQR), d & $5.00(2.50-11.00)$ \\
\hline Patients receiving mechanical ventilation during PSG, n (\%) & $28(54)$ \\
\hline Patients receiving an opioid, benzodiazepine or propofol, n (\%) & $43(81)$ \\
\hline Patients administered opioid and benzodiazepine medications ${ }^{9}, \mathrm{n}(\%)$ & $16(30)$ \\
\hline Patients administered benzodiazepine/propofol, n (\%) & $28(53)$ \\
\hline Patients administered opioids, n (\%) & $32(60)$ \\
\hline
\end{tabular}

${ }^{a}$ SD, standard deviation; ${ }^{b}$ APACHE, Acute Physiology and Chronic Health Evaluation; ${ }^{c}$ SOFA, Sequential Organ Failure Assessment; ${ }^{d}$ VICS, Vancouver Interaction Calmness Scale $\left(I=\right.$ interaction score $5-30$, higher scores are desirable, $\mathrm{C}=$ calmness score $5-30$, higher scores are desirable), ${ }^{e} \mathrm{BMI}$, body mass index; ${ }^{f} \mathrm{IQR}$, interquartile range; ${ }^{g}$ including propofol.

patients were extubated (that is, had their endotracheal tube removed) during PSG monitoring. The median TST of patients who received mechanical ventilation during PSG recording and those who did not was 05:14 (IQR: 03:36 to 07:57) vs. 03:57 (IQR: 01:39 vs. 06:47;

Table 2 Sleep outcomes: subjective reports

\begin{tabular}{ll}
\hline Outcomes & Patients $(\mathbf{n}=\mathbf{4 8})$ \\
$\mathrm{ISI}^{a}$ score, median $\left(\mathrm{IQR}^{b}\right)$ & $6.00(1.00-13.75)$ \\
$\mathrm{ISI}$ score $\geq 15, \mathrm{n}(\%)$ & $10(21)$ \\
Sleep quality pre-hospitalisation, median (IQR), 1-10 & $8.00(5.00-9.00)$ \\
& Patients $(\mathbf{n}=\mathbf{4 0})$ \\
Total RCSQ ${ }^{c}$ score in ICU, median (IQR), mm & $57.50(32.00-70.00)$ \\
& Patients $(\mathbf{n}=\mathbf{4 5})$ \\
Total RCSQ score in ward, median (IQR), mm & $57.40(36.70-74.40)^{d}$ \\
& Patients $(\mathbf{n}=\mathbf{4 3})$ \\
Sleep disturbing factors (SICQ $)$, mean (SD $)^{f}, 1-10$ & \\
Noise & $5.70(2.75)$ \\
Nursing interventions & $5.05(2.44)$ \\
Light & $5.15(2.61)$ \\
Diagnostic testing & $4.49(2.67)$ \\
Vital signs & $4.25(2.12)$ \\
Blood samples & $4.01(2.20)$ \\
Administration of medications & $3.84(2.12)$ \\
\hline
\end{tabular}

${ }^{a} \mathrm{ISI}$, Insomnia Severity Index; ${ }^{b} \mathrm{QQR}$, interquartile range = standard deviation; ${ }^{c} \mathrm{RCSQ}$, Richards Campbell Sleep Questionnaire; ${ }^{d}$ related samples Wilcoxon signed ranks test comparing total RCSQ ICU and ward scores $P=0.61,{ }^{e} \mathrm{SICQ}$, Sleep in Intensive Care Questionnaire; ${ }^{f} \mathrm{SD}$, standard deviation.
$P=0.049)$ and median arousal indices (20.50 IQR: 11.50 to 32.5 vs. 29.50 IQR: 22.00 to $40.87, P=0.018$ ). The differences for other sleep parameters did not reach statistical significance for patients who received mechanical ventilation during PSG recording and those who did not (for example, stage 1: 22.87 (24.13) vs. 27.54 (19.85) \%, stage 2: 69.99 (24.02) vs. 67.30 (20.17) \% and REM: 4.47 (5.90) vs. 2.38 (4.91) \%).

The mean equivalent dose of morphine was 12.46 (SD: $24.42) \mathrm{mcg} / \mathrm{kg} / \mathrm{h}$ and equivalent dose of midazolam was 2.26 (SD: 11.60$) \mathrm{mcg} / \mathrm{kg} / \mathrm{h}$. Six patients received temazepam (four received $20 \mathrm{mg}$ and two $10 \mathrm{mg}$ ) for night sedation. There was a difference in the median arousal indices for patients who received benzodiazepine medication/propofol and those who did not (22.00 IQR: 11.25 to 31.75 vs. 30.00 IQR: 20.75 to $40.75 ; P=0.019$ ). The differences for other sleep parameters did not reach statistical significance for patients who received benzodiazepine medication/propofol and those who did not (for example, stage 1: 26.41 (20.74) vs. 23.71 (23.75) \%, stage 2: 67.42 (19.38) vs. 69.97 (24.74) \% and REM: 2.85 (4.76) vs. 4.14 (6.16) \%).

\section{Discussion}

This study provides a current characterisation of the quality and quantity of sleep in intensive care patients, together with factors that potentially affect the sleep of patients in ICU. Sleep quality was poor; over $90 \%$ of patients' sleep was stage 1 and 2 and TST was below that experienced by 


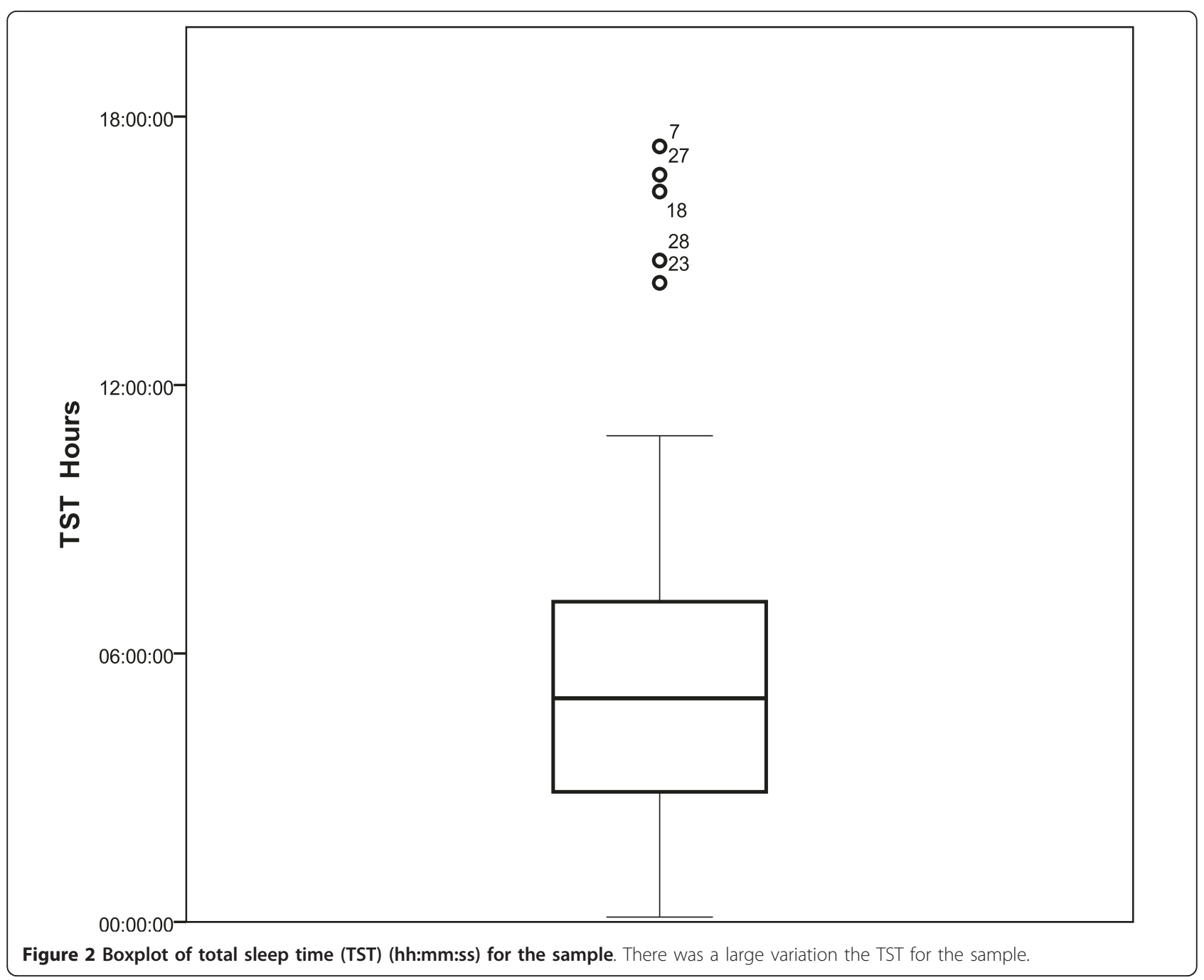

Table 3 Sleep outcomes: PSG-derived data, sleep time and stages

\begin{tabular}{ll}
\hline Outcomes & (n = 53) \\
Duration of PSG recording, median (IQR), hh:mm & $24: 00(23: 37-24: 00)$ \\
TST $^{a}$, median (IQR $\left.{ }^{b}\right)$, hh:mm & $05: 00(02: 52-07: 14)$ \\
Duration of sleep without waking, median (IQR), hh:mm & $00: 03(00: 02-00: 05)$ \\
Number of sleep periods, median (IQR) & $38.00(19.00-56.50)$ \\
Sleep during daytime hours, median (IQR), \% & $41(24-55)$ \\
Stage 1, median (IQR), hh:mm & $01: 00(00: 22-01: 31)$ \\
Stage 1, median (IQR), \% & $19(8-31)$ \\
Stage 2, median (IQR), hh:mm & $03: 03(01: 36-05: 19)$ \\
Stage 2, median (IQR), \% & $73(58-87)$ \\
Slow wave sleep, median (IQR), hh:mm & $00: 00(00: 00-00: 04)^{c}$ \\
Slow wave sleep, median (IQR), \% & $0(0-1)^{c}$ \\
REM ${ }^{d}$, median (IQR), hh:mm & $00: 00(00: 00-00: 21)^{e}$ \\
REM, median (IQR), \% & $0(0-6)^{e}$ \\
Arousals, median (IQR), No. per hr & $27.00(14.00-37.50)$ \\
\hline
\end{tabular}

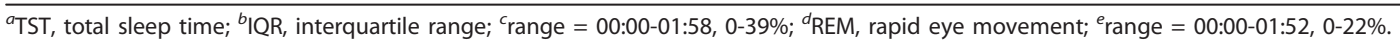


Table 4 Interrater reliability (Cohen's Kappa statistic) for Rechtschaffen and Kales scoring for sleep technologists (16 recordings)

\begin{tabular}{lll}
\hline Sleep stage/state & Technologists one and two, $\mathbf{1 8 , 6 4 4}$ epochs $\left(\mathbf{9 5 \%} \mathrm{Cl}^{\boldsymbol{a}}\right)$ & Technologists two and three, 25,908 epochs $\mathbf{( 9 5 \% ~} \mathbf{C l})$ \\
Stage 1 & $0.12(0.10-0.13)$ & $0.08(0.06-0.10)$ \\
Stage 2 & $0.58(0.46-0.72)$ & $0.55(0.54-0.56)$ \\
Stage 3/4 & $0.76(0.70-0.82)$ & $0.20(0.14-0.23)$ \\
REM $^{b}$ & $0.44(0.39-0.49)$ & $0.41(0.36-0.44)$ \\
Sleep/wake & $0.68(0.65-0.69)$ & $0.58(0.55-0.59)$ \\
\hline
\end{tabular}

${ }^{a} \mathrm{Cl}$, confidence interval; ${ }^{b} \mathrm{REM}$, rapid eye movement.

healthy adults. These results indicate that there has been no improvement since studies published between 1976 and 2003 [3,5,24-26], despite improvements in ICU design, technology and health care personnel training. Sleep fragmentation and unconventional architecture was evident with multiple short, non-contiguous sleep periods and numerous arousals. Arousals were frequent, as well as non-sequential stage changes [4,27]. A large percentage of TST was during daytime hours

Sound levels were elevated and exceeded World Health Organization (WHO) standards for hospitals, that is Leq should not $>35 \mathrm{~dB}(\mathrm{~A})$ in patient areas [28]. In our study, Leq was $>49 \mathrm{~dB}(\mathrm{~A})$. Continuous equivalent sound pressure levels were $10 \mathrm{~dB}(\mathrm{~A})$ lower than in many studies in ICU $[5,29,30]$ but similar to others $[26,31]$. The frequency of sound peaks $>80 \mathrm{~dB}(\mathrm{C})$ was notable (range: 31 to $1,436 / \mathrm{h}$ ) and high in comparison with other studies. Kahn et al. [32] reported a mean of $56 / \mathrm{h}$ before a noise reduction program and $40 / \mathrm{h}$ afterwards, while the mean reported by Meyer et al. [33] was $60 / \mathrm{h}$. It is somewhat surprising that there was no correlation between arousal indices and the number of sound peaks $>80 \mathrm{~dB}(\mathrm{~A})$, although it is possible that patients had become accustomed to high sound levels. Illuminance levels were appropriate at night (median $<2$ lux). However, daytime illuminance levels (74 lux) may have been too low to encourage a 24-hour circadian rhythm. Study of endogenous melatonin secretion in healthy participants indicates that illuminance levels of $<100$ lux may not be sufficiently bright to suppress melatonin secretion in some individuals [34]. Thus patients in our study may have had high melatonin levels during the day that contributed to the proportion of daytime sleep. The mean number of treatment/care events was $40(1.7 / \mathrm{h})$. This contrasts with hourly rates reported by others (for example 3,7 and $6 / h$ ) $[6,25,26]$. Underrecording by the bedside nurses may have occurred in our study. While an attempt was made to record care events/ treatment in a synchronous manner, this proved difficult in practice. Hence we are unable to draw conclusions about the relationship between such events and arousals.

Medications administered and considered to potentially affect sleep noted during PSG recording were mainly opioids and sedative medications. A large proportion
(60\%) of patients received opioid medications, potentially affecting their sleep. SWS has been found to be reduced by opioids, with a concomitant increase in stage 2 sleep [35]. A large percentage of patients in our study received benzodiazepines or propofol (53\%) (however, only lightly sedated patients were enrolled; the mean VICS score was 27.06 (SD: 3.80)). This may also have contributed to the high proportion of stage 2 and lack of REM sleep [36]. However, it is notable that patients who received benzodiazepine medication or propofol had fewer arousals than patients who did not. Despite the apparent suppression of SWS and REM, sleep may have been more consolidated when sedative medications were administered. The somewhat surprising findings that TST was higher and there were fewer arousal indices in patients who received mechanical ventilation during PSG recording may be partly attributable to the larger sedative medication doses they received (mean: 3.49 (SD: 15.64) vs. 0.40 (SD: 0.75) $\mathrm{mcg} / \mathrm{kg} / \mathrm{h}$ ).

Patients' self-reports of sleep quality in ICU were poor but comparable to previous studies. The mean RCSQ score from patients ready for transfer to the ward in the same ICU $(\mathrm{n}=222)$ was $47.18 \mathrm{~mm}$ [37]. In a study investigating the utility of the RCSQ and concordance of nurse and patient sleep assessment in ICU, the mean RCSQ score was $45.50 \mathrm{~mm}$ [38].

Patient perceptions of sources of sleep disruption were similar to previous studies. Noise was rated the most disruptive, as has been reported elsewhere [26]. In the development of the SICQ, ratings of disruptive activities were lower and noise (4.5) was less disruptive than vital signs (5.5) and phlebotomy (5.5) [10]. However, the SICQ was first reported in 1999; non-invasive vital signs monitoring and blood sampling in the current study would be expected to be less disruptive than techniques in use in the 1990s.

\section{Study strengths and limitations}

This study is the largest of its kind to be conducted using PSG with simultaneous data collection for factors known to affect sleep in ICU and is the first to present data from the Australian context. In addition, other investigators have rarely collected data on the patients' perception of 
sleep quality and potential sleep-disrupting factors in conjunction with PSG recording. This subjective information is vital in corroborating objective data, particularly as sleep is a subjective experience.

A limitation that became evident during the study was the difficulty in interpreting the PSG data using conventional $\mathrm{R}$ and $\mathrm{K}$ analysis. One patient's EEG waveform was affected with 'alpha intrusion' (alpha wave activity superimposed on delta waves), which made analysis impossible (the likely explanation being the administration of antipsychotic medications after enrolment/PSG recording began). Observation of EEG delta wave activity in some patients who appeared to be awake has been noted in other studies $[4,5,39]$. Challenges in scoring ICU patients' sleep data were recently reported by Drouot et al. [39] in their analysis of PSG data from two studies conducted previously. The investigators noted the presence of EEG delta wave activity during apparent wakefulness (the presence of EMG, EOG and limb activity) and a lack of $\mathrm{K}$ complexes and sleep spindles preventing classification of stage 2 . However, interrater and intrarater scorer reliability checks were not performed in that study.

Another limitation is the effect of benzodiazepine medications on the interpretation of sleep parameters. It is known that benzodiazepine medication increases EEG beta wave activity and reduces EEG delta wave activity [40]. Benzodiazepine medication also increases EEG spindle activity [41] (though this appears not to have been the case in our study). The combined effect of the opioids and sedative medication on EEG activity likely affected the results. However, since these medications are essential adjuncts in the treatment and comfort of many ICU patients, excluding patients who received them would have severely limited recruitment and the generalisability of our results. Other limitations were the presence of factors such as the use of different modes of mechanical ventilation and health conditions such as systemic inflammatory response, but such factors are common in ICU patients and their exclusion would also limit the applicability of the results to the ICU patient population.

Patient enrolment was limited by a number of factors. The availability of PSG monitoring equipment and the principal researcher were the primary factors for patients being discharged before they were approached to participate. For this study, we had access to only two people trained in sleep recordings (RE and MF) and one portable sleep-monitoring device, creating practical limitations to the number and frequency of studies performed. In addition, there was often limited opportunity in which patients were cognitively able to agree to participate before they were discharged to the hospital ward. A large proportion of patients approached about the study declined to participate; many considered the application of monitoring as a set-back in their condition regardless of the reason for it. This may limit the generalisability of the results to a subset of patients treated in the study ICU.

Interrater reliability of the $\mathrm{R}$ and $\mathrm{K}$ analysis was moderate, 0.56 for sleep technologists one and two and 0.51 for sleep technologists two and three but lower than Kappa values for sleep technologists in sleep investigation units (for example 0.72 [42]). Interrater reliability for each stage was also lower than in sleep laboratory studies. The reliability of PSG analysis in ICU patients has been infrequently reported. In one study of critically ill, non-ventilated trauma patients' night-time sleep, interrater reliability for one overnight recording, was reported as Kappa $=0.82$ [43]. However, in a comparison by Ambrogio et al. of four methods of analysing ICU patients' PSG data, interrater reliability was considerably lower than in the present study $($ Kappa $=0.19)[44]$.

\section{Conclusions}

The quantity and quality of patients' sleep while in this ICU using both objective and subjective assessment methods were found to be poor. Given the similarity between the sleep outcomes and prevalence of potential sleep-disturbing factors in our study and previous studies, sleep disruption clearly remains a substantial problem for many ICU patients. Importantly, the current study has added to evolving knowledge of the challenges of using PSG in ICU and analysing the data. An alternative objective sleep assessment method is required for ICU patients in order to further our understanding of sleep disruption in this vulnerable patient population and to test clinical interventions for their well-being and recovery.

\section{Key messages}

-Sleep in ICU patients is highly fragmented with concomitant deficiencies in SW and REM sleep.

.Use of PSG in ICU patients and analysing the data are challenging.

.There is a need to develop alternative methods to conventional PSG staging to measure sleep in ICU patients and develop interventions which will improve sleep.

\footnotetext{
Abbreviations

APACHE, Acute Physiology and Chronic Health Evaluation; ECG, electrocardiograph; EEG, electroencephalograph; EMG, electromyograph; EOG, electrooculograph; ICU, intensive care unit; ISI, Insomnia Severity Index; Leq, equivalent sound pressure levels; Lpeak, peak sound pressure levels; PCV, pressure control ventilation; PSG, polysomnography; $R$ and $K$, Rechtschaffen and Kales; RASS, Richmond Agitation Sedation Scale; RCSO Richards-Campbell Sleep Questionnaire; REM, rapid eye movement; RN, registered nurse; SICQ, Sleep in Intensive Care Questionnaire; SIMV, synchronised intermittent ventilation; SOFA, Sequential Organ Failure Assessment; SWS, slow wave sleep; TST, total sleep time; VAS, visual analogue scale; VICS, Vancouver Interaction Scale.
} 


\section{Authors' contributions}

RE conceived and developed the protocol and design, acquired and analysed the data, wrote the manuscript, SM and PC supervised RE, assisted with the development of the design, interpretation of the data and writing the manuscript and MF assisted with protocol development, data acquisition and writing the manuscript. All the authors have read and approved the manuscript for publication.

\section{Competing interests}

The authors declare that they have no competing interests.

\section{Acknowledgements}

The authors thank the Intensive Care Foundation, Australian College of Critical Care Nurses, the Skipper family (Royal North Shore Hospital Nursing Research Scholarship), the Northcare Foundation, Pink Ladies Committee (Royal North Shore Hospital), Faculty of Engineering and Information Technology (University of Technology Sydney) and the patients and health care personnel for their generous contributions to the study.

\section{Author details}

'Faculty of Health, University of Technology Sydney, 235-253 Jones Street, Broadway 2007, New South Wales, Australia. ${ }^{2}$ Intensive care unit, Royal North Shore Hospital, Northern Sydney Local Health District, Reserve Road, St Leonards 2065, New South Wales, Australia. ${ }^{3}$ Faculty of Medicine, University of Sydney, Fisher Road, Camperdown 2006 New South Wales, Australia. ${ }^{4}$ Centre for Sleep Health \& Research, Royal North Shore Hospital, Reserve Road, St Leonards 2065, New South Wales, Australia. ${ }^{5}$ Musculoskeletal Network, Agency for Clinical Innovation, 67 Albert Avenue, Chatswood 2067, New South Wales, Australia.

\section{Received: 23 November 2012 Revised: 17 January 2013}

Accepted: 14 March 2013 Published: 18 March 2013

\section{References}

1. Gallicchio L, Kalesan : Sleep duration and mortality: a systematic review and meta-analysis. J Sleep Res 2009, 18:148-158, Epub 2009 Aug 4.

2. Grandner MA, Hale L, Moore M, Patel NP: Mortality associated with short sleep duration: the evidence, the possible mechanisms, and the future. Sleep Med Rev 2010, 14:191-203, Epub 2009 Nov 26.

3. Aurell J, Elmqvist D: Sleep in the surgical intensive care unit: continuous polygraphic recording of sleep in nine patients receiving postoperative care. Br Med J (Clin Res Ed) 1985, 290:1029-1032, Epub 1985 Apr 6.

4. Cooper AB, Thornley KS, Young GB, Slutsky AS, Stewart TE, Hanly PJ: Sleep in critically ill patients requiring mechanical ventilation. Chest 2000, 117:809-818, Epub 2000 Mar 14.

5. Freedman NS, Gazendam J, Levan L, Pack Al, Schwab RJ: Abnormal sleep/ wake cycles and the effect of environmental noise on sleep disruption in the intensive care unit. Am J Respir Crit Care Med 2001, 163:451-457, Epub 2001 Feb 17.

6. Hardin KA, Seyal M, Stewart T, Bonekat HW: Sleep in critically ill chemically paralyzed patients requiring mechanical ventilation. Chest 2006 129:1468-1477, Epub 2006 June 17.

7. Aaron JN, Carlisle CC, Carskadon MA, Meyer TJ, Hill NS, Millman RP: Environmental noise as a cause of sleep disruption in an intermediate respiratory care unit. Sleep 1996, 19:707-710.

8. Bosma K, Ferreyra G, Ambrogio C, Pasero D, Mirabella L, Braghiroli A, Appendini L, Mascia L, Ranieri VM: Patient-ventilator interaction and sleep in mechanically ventilated patients: pressure support versus proportional assist ventilation. Crit Care Med 2007, 35:1048-54, Epub 2007 March 6.

9. Nicolas A, Aizpitarte E, Iruarrizaga A, Vazquez M, Margall A, Asiain C: Perception of night-time sleep by surgical patients in an intensive care unit. Nurs Crit Care 2008, 13:25-33, Epub 2008 Jan 30

10. Freedman NS, Kotzer N, Schwab RJ: Patient perception of sleep quality and etiology of sleep disruption in the intensive care unit. Am J Respir Crit Care Med 1999, 159:1155-1162, Epub 1999 Apr 8.

11. Bastien $\mathrm{CH}$, Vallieres $\mathrm{A}$, Morin $\mathrm{CM}$ : Validation of the Insomnia Severity Index as an outcome measure for insomnia research. Sleep Med 2001, 2:297-307, Epub 2001 July 5.

12. Jasper $\mathrm{HH}$ : Report of the committee on methods of clinical examination in electroencephalography: 1957. Electroencephalogr Clin Neurophysiol 1958, 10:370-375.
13. Elliott RM, McKinley SM, Eager D: A pilot study of sound levels in an Australian adult general intensive care unit. Noise Health 2010, 12:26-36, Epub 2010 Feb 18

14. Richards KC, O'Sullivan PS, Phillips RL: Measurement of sleep in critically ill patients. J Nurs Meas 2000, 8:131-144, Epub 2001 Mar 3.

15. Richards KC, Bairnsfather L: A description of night sleep patterns in the critical care unit. Heart Lung 1988, 17:35-42, Epub 1988 Jan 1.

16. Australian and New Zealand Intensive Care Society: Appendix C: ICU Diagnosis APACHE III. ANZICS Adult patient database data dictionary 2004, 95-97.

17. Vincent JL, Moreno R, Takala J, Willatts S, De Mendonca A, Bruining H, Reinhart CK, Suter PM, Thijs LG: The SOFA (Sepsis-related Organ Failure Assessment) score to describe organ dysfunction/failure. On behalf of the Working Group on Sepsis-Related Problems of the European Society of Intensive Care Medicine. Intensive Care Med 1996, 22:707-710, Epub 1996 July 1.

18. Rechtschaffen A, Kales A: A manual of standardized terminology: techniques and scoring system for sleep stages of human subjects Los Angeles: UCLA Brain Information Service/Brain Research Institute; 1968.

19. Iber C, Ancoli-Israel S, Chesson A, Quan SF: In AASM manual for the scoring of sleep and associated events: rules, terminology and technical specification.. 1 edition. Edited by: Iber C, Ancoli-Israel S, Chesson A, Quan SF. Westchester: American Academy of Sleep Medicine; 2007:

20. Ballantyne J, Bonica JJ, Fishman S: In Bonica's management of pain. 4 edition. Edited by: Fishman SM, Ballantyne JC, Rathmell JP. Philadelphia: Lippincott Williams 2009:

21. Ashton $\mathrm{CH}$ : Guidelines for the rational use of benzodiazepines. Drugs 1994, 48:25-40

22. de Lemos J, Tweeddale M, Chittock D: Measuring quality of sedation in adult mechanically ventilated critically ill patients: the Vancouver Interaction and Calmness Scale. J Clin Epidemiol 2000, 53:908-919.

23. Sessler CN, Gosnell MS, Grap MJ, Brophy GM, O'Neal PV, Keane KA, Tesoro EP, Elswick RK: The Richmond Agitation-Sedation Scale: validity and reliability in adult intensive care unit patients. Am I Respir Crit Care Med 2002, 166:1338-1344, Epub 2002 Nov 15.

24. Edell-Gustafsson UM, Hetta JE, Aren CB: Sleep and quality of life assessment in patients undergoing coronary artery bypass grafting J Adv Nurs 1999, 29:1213-1220, Epub 1999 May 13.

25. Hilton BA: Quantity and quality of patients' sleep and sleep-disturbing factors in a respiratory intensive care unit. J Adv Nurs 1976, 1:453-468, Epub 1976 Nov 1.

26. Gabor JY, Cooper AB, Crombach SA, Lee B, Kadikar N, Bettger HE, Hanly PJ: Contribution of the intensive care unit environment to sleep disruption in mechanically ventilated patients and healthy subjects. Am J Respir Crit Care Med 2003, 167:708-715, Epub 2003 Feb 25.

27. Friese RS, Diaz-Arrastia R, McBride D, Frankel H, Gentilello LM: Quantity and quality of sleep in the surgical intensive care unit: are our patients sleeping? J Trauma 2007, 63:1210-1214, Epub 2008 Jan 24.

28. Berglund B, Lindvall T: Guidelines for community noise Geneva, Switzerland: World Health Organization; 1999.

29. Wallace CJ, Robins J, Alvord LS, Walker JM: The effect of earplugs on sleep measures during exposure to simulated intensive care unit noise. Am J Crit Care 1999, 8:210-219, Epub 1999 July 7.

30. Hilton BA: Noise in acute patient care areas. Res Nurs Health 1985, 8:283-291, Epub 1985 Sept 1.

31. Blomkvist V, Eriksen CA, Theorell T, Ulrich R, Rasmanis G: Acoustics and psychosocial environment in intensive coronary care. Occup Environ Med 2005, 62:e1, Epub 2005 Feb 23.

32. Kahn DM, Cook TE, Carlisle CC, Nelson DL, Kramer NR, Millman RP: Identification and modification of environmental noise in an ICU setting. Chest 1998, 114:535-540, Epub 1998 Sept 3.

33. Meyer TJ, Eveloff SE, Bauer MS, Schwartz WA, Hill NS, Millman RP: Adverse environmental conditions in the respiratory and medical ICU settings. Chest 1994, 105:1211-1216, Epub 1994 Apr 01.

34. Gronfier C, Wright KP Jr, Kronauer RE, Czeisler CA: Entrainment of the human circadian pacemaker to longer-than-24-h days. Proc Natl Acad Sci U S A 2007, 104:9081-9086, Epub 2007 May 16.

35. Dimsdale JE, Norman D, DeJardin D, Wallace MS: The effect of opioids on sleep architecture. J Clin Sleep Med 2007, 3:33-36, Epub 2007 June 15.

36. Monti MJ, Monti D: Overview of currently available benzodiazepine and nonbenzodiazepine hypnotics. In Clinical Pharmacology of Sleep. 
Edited by: Pandi-Perumal SR, Monti JM. Basel, Switzerland: Birkhauser Verlag; 2006:207-223.

37. Intensive care patients report that their sleep is poor. In In Proceedings of the 36th Australian and New Zealand Intensive Care Society and Australian College of Critical Care Nurses Annual Scientific Meeting (ASM) on Intensive Care. Edited by: McKinley S, Elliott R, Elliott D, Fien M. Brisbane, Australia; 2011.

38. Frisk U, Nordstrom G: Patients' sleep in an intensive care unit - patients' and nurses' perception. Intensive Crit Care Nurs 2003, 19:342-349, Epub 2003 Nov 26.

39. Drouot X, Roche-Campo F, Thille AW, Cabello B, Galia F, Margarit L, d'Ortho MP, Brochard L: A new classification for sleep analysis in critically ill patients. Sleep Med 2012, 13:7-14, Epub 2011 Dec 14.

40. Bastien CH, LeBlanc M, Carrier J, Morin CM: Sleep EEG power spectra, insomnia, and chronic use of benzodiazepines. Sleep 2003, 26:313-317, Epub 2003 May 17.

41. Aeschbach D, Dijk DJ, Trachsel L, Brunner DP, Borbely AA: Dynamics of slow-wave activity and spindle frequency activity in the human sleep EEG: effect of midazolam and zopiclone. Neuropsychopharmacology 1994, 11:237-244, Epub 1994 Dec 1.

42. Danker-Hopfe H, Anderer P, Zeithofer J, Boeck M, Dorn H, Gruber G, Heller E, Loretz E, Moser D, Parapatics S, Saletu B, Schmidt A, Dorffner G: Interrater reliability for sleep scoring according to the Rechtschaffen \& Kales and the new AASM standard. J Sleep Res 2009, 18:74-84, Epub 2009 Mar 3.

43. Fontaine DK: Measurement of nocturnal sleep patterns in trauma patients. Heart Lung 1989, 18:402-410, Epub 1989 July 1.

44. Ambrogio C, Koebnick J, Quan SF, Ranieri M, Parthasarathy S: Assessment of sleep in ventilator-supported critically III patients. Sleep 2008, 31:1559-1568, Epub 2008 Nov 19

doi:10.1186/cc12565

Cite this article as: Elliott et al:: Characterisation of sleep in intensive care using 24-hour polysomnography: an observational study. Critical Care 2013 17:R46.

\section{Submit your next manuscript to BioMed Central} and take full advantage of:

- Convenient online submission

- Thorough peer review

- No space constraints or color figure charges

- Immediate publication on acceptance

- Inclusion in PubMed, CAS, Scopus and Google Scholar

- Research which is freely available for redistribution 